\title{
Photoluminescence measurements of quantum-dot-containing semiconductor microdisk resonators using optical fiber taper waveguides
}

\author{
Kartik Srinivasan, ${ }^{1, *}$ Andreas Stintz, ${ }^{2}$ Sanjay Krishna, ${ }^{2}$ and Oskar Painter ${ }^{1}$ \\ ${ }^{1}$ Thomas J. Watson, Sr., Laboratory of Applied Physics, California Institute of Technology, Pasadena, California 91125, USA \\ ${ }^{2}$ Center for High Technology Materials, University of New Mexico, Albuquerque, New Mexico 87101, USA \\ (Received 11 June 2005; revised manuscript received 11 August 2005; published 10 November 2005)
}

\begin{abstract}
Optical fiber taper waveguides are used to improve the efficiency of room temperature photoluminescence measurements of AlGaAs microdisk resonant cavities with embedded self-assembled InAs quantum dots. As a near-field collection optic, the fiber taper improves the collection efficiency from microdisk lasers by a factor of 15-100 times in comparison to conventional normal incidence free-space collection techniques. In addition, the fiber taper can serve as an efficient means for pumping these devices, and initial measurements employing fiber pumping and collection are presented. Implications of this work towards chip-based cavity quantum electrodynamics experiments are discussed.
\end{abstract}

DOI: 10.1103/PhysRevB.72.205318

PACS number(s): 42.60.Da, 42.50.Pq, 42.70.Qs

\section{INTRODUCTION}

The ability to efficiently couple light into and out of semiconductor microcavities is an important aspect of many microphotonic technologies, ${ }^{1}$ and plays a vital role in chipbased implementations of cavity quantum electrodynamics (cQED) for quantum networking and cryptography. ${ }^{2-4}$ While some geometries, such as micropillar cavities, exhibit highly directional emission that can be effectively collected, ${ }^{5,6}$ coupling to wavelength-scale semiconductor microcavities is in general nontrivial, ${ }^{4,7-9}$ due to a number of factors. These include the size disparity between the modes of the microcavity and those of standard free-space and fiber optics, the refractive index difference between semiconductors and glass or air, and the potentially complicated cavity mode profiles sustained by these devices. One technique that we have recently employed to couple efficiently to semiconductor microcavities is evanescent coupling through an optical fiber taper waveguide. ${ }^{10}$ The fiber taper ${ }^{11,12}$ is simply a standard single mode fiber that has been heated and stretched down to a minimum diameter on the order of a wavelength. Such fiber tapers have been used as near-ideal coupling channels for glass-to-glass coupling with silica-based microcavities such as microspheres ${ }^{13-16}$ and microtoroids. ${ }^{17}$ Our recent experiments have indicated that they can also serve as efficient couplers to high-refractive index semiconductor-based devices, such as photonic crystal waveguides, ${ }^{18}$ photonic crystal cavities, ${ }^{10,19}$ and microdisks. ${ }^{20,21}$ Here, we consider the use of fiber tapers within active semiconductor devices consisting of AlGaAs microdisk cavities with embedded InAs quantum dots. While our previous work on microdisks within this material system primarily utilized fiber tapers for passive measurements such as the characterization of cavity quality factors, ${ }^{21}$ in this work, we focus on using the fiber taper as an efficient coupler for injecting pump light into and extracting the light emitted by semiconductor quantum dots into microdisk whispering gallery modes. The immediate device application that we study here are room-temperature, fiber-coupled microdisk-quantum-dot lasers, but the results described are directly applicable to future studies of cQED phenomena in semiconductor-based systems.
In Sec. II, we qualitatively describe the issues addressed in this paper, as well as the methods used in device fabrication and the experimental setup we use. In Sec. III, we briefly review passive measurements in the $1200 \mathrm{~nm}$ wavelength band to determine the intrinsic optical losses of the optical resonant cavities under study. In Sec. IV, we present experimental results demonstrating the improvements that result when free-space collection is replaced by fiber-based collection in photoluminescence measurements, while in Sec. V, we present initial results on microdisk lasers that employ both fiber pumping and fiber collection. Finally, in Sec. VI, we consider some of the applications of this work to future experiments.

\section{PRELIMINARY DISCUSSION AND EXPERIMENTAL METHODS}

The specific devices we consider are AlGaAs/GaAs microdisk cavities with embedded quantum dots (QDs). The epitaxy used is shown in Table I, and consists of a single layer of InAs quantum dots embedded in an InGaAs quantum well, ${ }^{22}$ which is in turn sandwiched between layers of AlGaAs and GaAs to create a $255 \mathrm{~nm}$ thick waveguide. This

TABLE I. Epitaxy for 1-DWELL microcavity lasers.

\begin{tabular}{ccc}
\hline \hline Layer & Materials & Thickness \\
\hline Surface cap layer & $\mathrm{GaAs}_{0.30}$ & $100 \AA$ \\
Top waveguide layer & $\mathrm{Al}_{0.30} \mathrm{Ga}_{0.70} \mathrm{As}$ & $400 \AA$ \\
Top waveguide layer & $\mathrm{GaAs}$ & $740 \AA$ \\
Quantum well layer & $\mathrm{In}_{0.15} \mathrm{Ga}_{0.85} \mathrm{As}$ & $60 \AA$ \\
Quantum dot layer & $\mathrm{InAs}$ & 2.4 monolayer \\
Barrier layer & $\mathrm{In}_{0.15} \mathrm{Ga}_{0.85} \mathrm{As}$ & $10 \AA$ \\
Bottom waveguide layer & $\mathrm{GaAs}_{0.30}$ & $740 \AA$ \\
Bottom waveguide layer & $\mathrm{Al}_{0.30} \mathrm{Ga}_{0.70} \mathrm{As}$ & $500 \AA$ \\
Sacrificial buffer layer & $\mathrm{Al}_{0.70} \mathrm{Ga}_{0.30} \mathrm{As}$ & $15000 \AA$ \\
Substrate & $\mathrm{GaAs}$ & $\mathrm{N} / \AA$ \\
\hline \hline
\end{tabular}




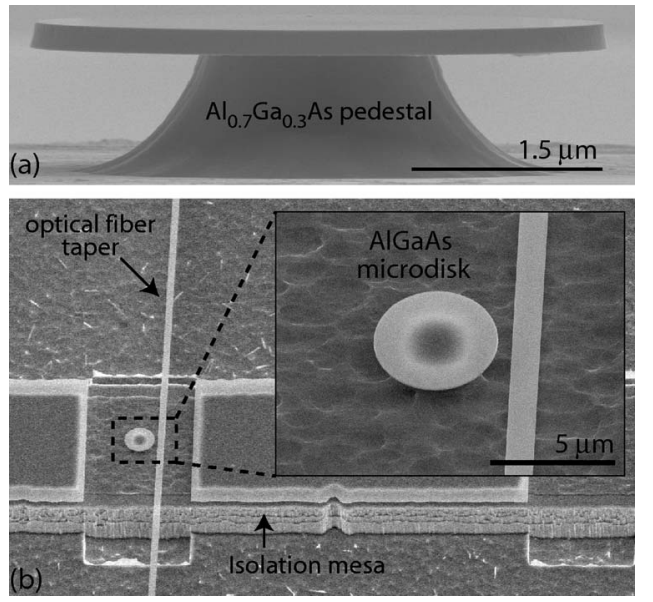

FIG. 1. (a) and (b) Scanning electron microscope (SEM) images of fabricated microdisk structures. Image (b) shows the isolation mesa that is incorporated in order to aid in the taper testing. An optical fiber taper aligned to the side of a microdisk is also visible in this image.

DWELL (short for dot-in-a-well) material has a room temperature ground state emission peak at around $1190 \mathrm{~nm}$ [Fig. $3(\mathrm{a})]$, and is grown on top of a $1.5 \mu \mathrm{m} \mathrm{Al}_{0.70} \mathrm{Ga}_{0.30} \mathrm{As}$ layer that eventually serves as a support pedestal for the microdisk. Fabrication of the microdisks (Fig. 1) is accomplished through the following series of steps: (i) Deposition of a $200 \mathrm{~nm} \mathrm{Si} \mathrm{N}_{y}$ mask layer, (ii) electron-beam lithography and a subsequent reflow of the resist, (iii) inductively coupled plasma reactive ion etch (ICP-RIE) of the $\mathrm{Si}_{x} \mathrm{~N}_{y}$ layer, (iv) ICP-RIE of the QD-containing waveguide layer, (v) photolithography and isolation of the microdisk onto a mesa stripe that is several microns above the rest of the chip [Fig. 1(b)], and (vi) HF acid etch of the $\mathrm{Al}_{0.70} \mathrm{Ga}_{0.30}$ As layer to form the pedestal which supports the disk. The fabricated microdisks in this work are $D \sim 4.5 \mu \mathrm{m}$ in diameter.

The free-space collection from a whispering gallery mode (WGM) of a microdisk is a function of a number of factors, including the position and numerical aperture (NA) of the collection lens, and the radiation pattern and quality factor $(Q)$ of the resonant mode. Optical losses from the microdisk include not only the (ideal) radiation due to radial tunneling of light from the disk periphery, but also scattering losses due to surface roughness imperfections at the disk edge and material absorption. For high-refractive index $(n \sim 3.5)$ III-V semiconductor microdisks, surface roughness scattering is typically the dominant form of radiation from the microcavity. The intrinsic radiation loss of semiconductor microdisks is almost negligible in all cases, save the smallest of microdisks; the radiation $Q$ of the lowest radial order WGM of the $D \sim 4.5 \mu \mathrm{m}$ microdisks studied here is greater than $10^{14}$ at the QD emission wavelength of $1200 \mathrm{~nm}$, and is greater than $10^{6}$ for $D \sim 2.0 \mu \mathrm{m}$. As such, any light that is collected through free-space methods is the result of scattering of the WGM off imperfections in the microdisk, ${ }^{8}$ a relatively inefficient and nondirectional process. Bulk material absorption and absorption due to surface states may also play a role, particularly when considering devices in which the $Q$ due to surface scattering can be in excess of $10^{5}$. This results in a situation where the more perfect the microdisk is made (through reduction in surface roughness), and the further the $Q$ factor is improved, the more difficult it becomes to collect light from the resonant modes. Although there may be some potential in modifying the disk geometry ${ }^{4,8}$ to improve this situation (for example, by etching a shallow second-order grating in the microdisk surface), the ability to do this while maintaining high $Q$ factors could be of potential difficulty. The most successful method to date for increasing collection efficiency from semiconductor microdisk resonators seems to be placement of the collection optics in the plane of the disk, ${ }^{23,24}$ resulting in more effective capture of the predominantly low-angle scattered light.

The fiber taper offers an attractive alternative because it provides a means to directly couple light out of the WGMs, without relying upon the weak intrinsic radiation of the microdisk or the non-directionality of surface roughness scattering. This evanescent near-field coupling, which is a function of the integrated modal overlap of the microdisk and taper modes over the interaction region, ${ }^{25,26}$ has been demonstrated to be appreciable in previous works with small diameter semiconductor microdisks ${ }^{20,21}$ where phasematching between the glass fiber taper waveguide and the semiconductor microdisk is not as limiting. While the fiber taper does load the cavity mode, and thus degrade its $Q$, the key point is that the added loss is primarily good loss in the sense that it can be efficiently collected into the taper mode of interest. ${ }^{16,19}$ This allows for the loaded $Q$ to be maintained at a high value while simultaneously obtaining high collection efficiency. ${ }^{20,21}$ The situation is analogous to the case of a Fabry-Perot cavity where one mirror is intentionally made to have a slightly lower reflectivity for output coupling, which limits the $Q$ of the cavity, but not beyond some acceptable level. While in that case, the cavity $Q$ is fixed by the mirror reflectivities, here we have some flexibility over the $Q$ and the amount of loading by adjusting the cavity-taper separation.

To compare free-space and fiber-taper-based collection, we use the experimental setup depicted in Fig. 2, which consists of a fiber taper probing station that has been incorporated into a standard photoluminescence (PL) measurement setup (refer to the Appendix for a detailed description). This integrated setup allows for a number of different measurements to be made. Passive measurements of the microdisk resonant modes are performed by sending a tunable laser into the taper's input and monitoring the wavelength-dependent transmission at the taper's output. Photoluminescence measurements can be done in any of four potential configurations (i) free space pumping, free space collection: Here, the fiber taper plays no role, and the vertically emitted power from the disks is collected into a multimode optical fiber that is then fed into the optical spectrum analyzer (OSA); (ii) free space pumping, fiber taper collection: Here, the output of the fiber taper is connected to the OSA; (iii) fiber taper pumping, free space collection: Here, the input of the fiber taper is connected to a fiber-coupled pump laser; (iv) fiber taper pumping, fiber taper collection: Here, the free-space optics used in the standard PL measurements play no role. 
(a)

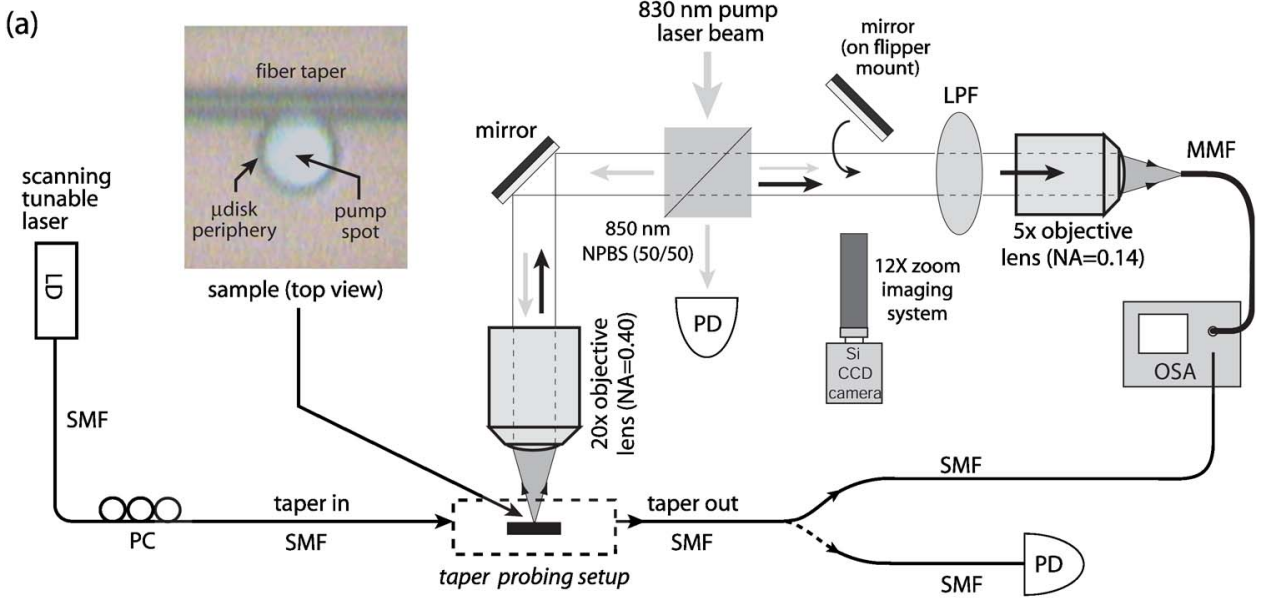

(b)

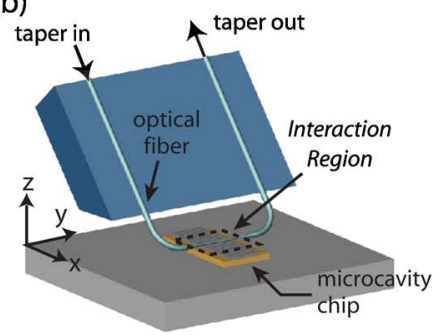

(c)

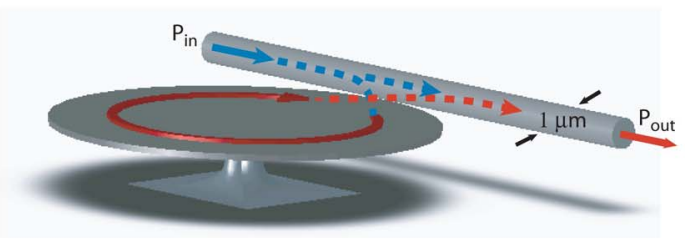

FIG. 2. (Color online) (a) Experimental setup for studying the QD-microdisk devices (see Appendix for more details), where the pump laser and photoluminescence beams are shown as light gray and black arrows, respectively. The inset shows a fiber taper aligned to a microdisk that is being optically pumped from the top. Acronyms used in the diagram: polarization controller $(\mathrm{PC})$, numerical aperture (NA), nonpolarizing beamsplitter (NPBS), photodetector (PD), long-pass pump rejection filter (LPF), single mode fiber (SMF), multimode fiber (MMF), optical spectrum analyzer (OSA). (b) Schematic of the fiber taper probing geometry. (c) Schematic of the taper-tomicrodisk interaction region, showing the resonant fiber taper coupling to WGMs of the microdisk.

\section{MEASUREMENT OF CAVITY $Q$ IN THE 1200 NM WAVELENGTH BAND}

The devices studied in this work have been previously characterized in the $1400 \mathrm{~nm}$ band, and $Q \mathrm{~s}$ as high as $3.6 \times 10^{5}$ have been measured. ${ }^{21}$ Those measurements were done at wavelengths significantly red-detuned from the QD emission band, where QD absorption and material absorption in the GaAs/AlGaAs waveguide layers are expected to be quite small. To confirm that the cavity $Q$ s are still high near the ground-state QD emission wavelength [peaked near $1190 \mathrm{~nm}$ as shown in Fig. 3(a)], we perform passive fibertaper-based measurements ${ }^{10,20,21}$ in the $1200 \mathrm{~nm}$ band. ${ }^{50}$ The input of the taper is connected to a fiber-coupled scanning tunable laser that has a wavelength range of $1215-1265 \mathrm{~nm}$ and is sampled at a resolution of $\sim 0.1 \mathrm{pm}$ (well above the laser's linewidth of $<5 \mathrm{MHz}$ ). The transmission past the microdisk is monitored by connecting the taper output to a fiber-pigtailed photodetector. The taper-WGM coupling is sensitive to polarization, and here coupling was optimized for TE-like WGMs with electric field polarized predominantly in the plane of the microdisk. The fiber taper is brought into the near-field of the microdisk by first vertically aligning the fiber taper within the plane of the microdisk and then bringing it in from the side towards the disk edge [Fig. 2(c)]. Resonances begin to appear within the taper's transmission spectrum when it is several hundred nanometers to the side of the microdisk. The high- $Q$ resonances within the transmission spectrum [Fig. 3(b)], under closer inspection, are seen to consist of a pair resonances dips [inset to Fig. 3(b)]. The two resonance dips (doublets) correspond to standing wave modes that are formed when surface scattering couples and splits the initially degenerate clockwise and counterclockwise traveling wave WGMs of the microdisk.
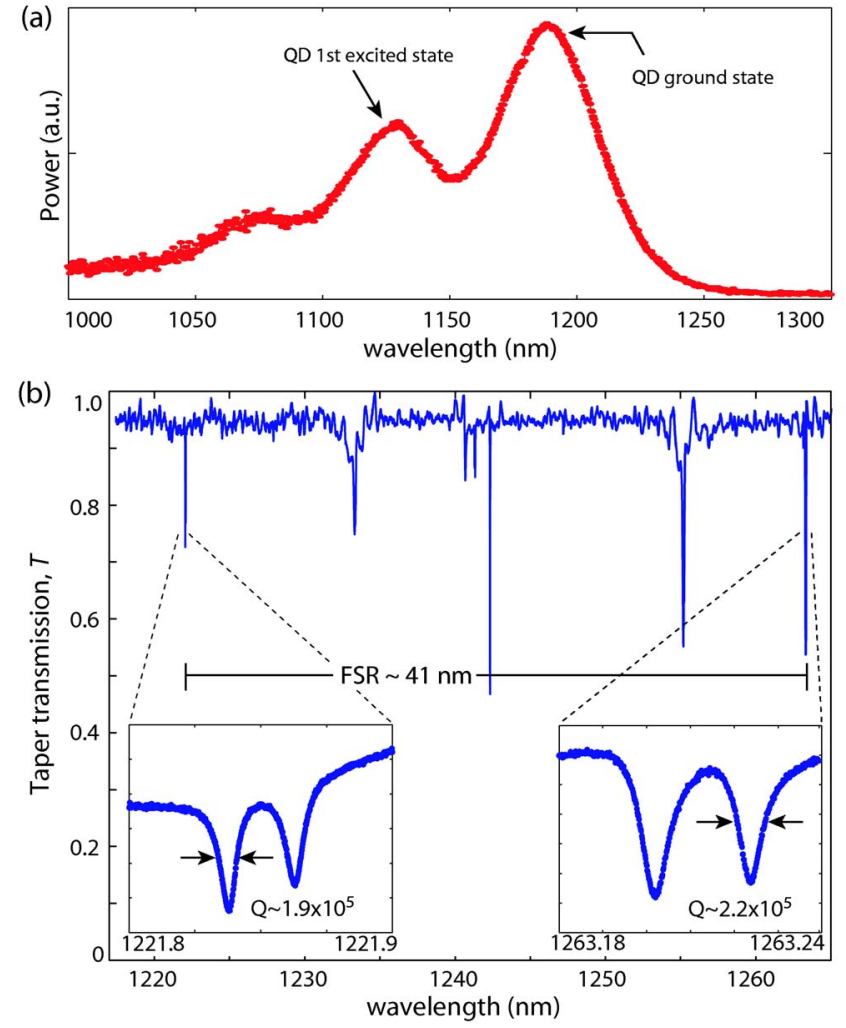

FIG. 3. (Color online) (a) Photoluminescence from an unprocessed region of the 1DWELL material. (b) Passive taper-based measurements of a microdisk in the $1200 \mathrm{~nm}$ band. The insets show high resolution scans for two sets of doublet modes for this device. These high resolution scans are taken when the taper-microdisk separation is a few hundred $\mathrm{nm}$ and the depth of coupling is $\sim 5 \%-10 \%$. 
Such doublet resonances have been observed by a number of authors for different whispering-gallery geometries and materials. $^{20,21,27,28}$

Finite-element frequency mode solutions ${ }^{29}$ of the WGM resonances of the microdisks studied in this work $(D=4.5 \mu \mathrm{m})$ show that the free spectral range (FSR) is $\sim 40 \mathrm{~nm}$ in the $1200 \mathrm{~nm}$ wavelength band for TE-polarized modes of low radial mode number $(q=1-4)$. Higher radial order WGMs $(q \geqslant 5)$ are expected to show up only very weakly in the fiber taper transmission owing to their small radiation limited Q factors $\left(\leqslant 10^{4}\right)$ and significantly larger overlap with the support pedestal. From the broad spectral wavelength scan shown in Fig. 3(b), a pair of deeply coupled resonant modes separated by a full FSR are observed $(\lambda \sim 1222$ and $1263 \mathrm{~nm})$, as well as several other deeply coupled resonant modes. Due to the extended nature of the higher order radial modes and their better phase-matching to a low-index glass waveguide such as the fiber taper, the coupling to the lowest order $q=1$ WGM is typically lower than that of the $q=2$ mode for similar sized microdisks. ${ }^{20,26} \mathrm{We}$ believe that these doublet modes at $\lambda \sim 1222 \mathrm{~nm}$ and $\lambda \sim 1263 \mathrm{~nm}$ are first order $(q=1)$ radial modes, while the mode at $\lambda \sim 1242 \mathrm{~nm}$ is probably a $q=2$ radial mode. The broader and more weakly coupled intermediate modes are most likely higher order radial modes, $q=3$, 4 (higher order slab modes in the vertical direction of microdisk are also a possibility, though less likely due to their reduced radiation $Q$ ). Examining the linewidth of the doublet resonances when the taper is relatively far away from the microdisk gives an estimate for the cold-cavity, unloaded $Q$ of the modes. $Q$ s as high as $2.2 \times 10^{5}$ at $\sim 1260 \mathrm{~nm}$ and as high as $1.9 \times 10^{5}$ at $\sim 1220 \mathrm{~nm}$ are measured in these microdisks [insets of Fig. $3(\mathrm{~b})$ ], the latter of which is only $30 \mathrm{~nm}$ red-detuned from the peak of the QD emission spectrum [Fig. 3(a)]. These $Q$ factors are still quite high for a wavelength-scale AlGaAs microcavity, ${ }^{23,24,30-32}$ and correspond to a cavity decay rate of $\kappa / 2 \pi \sim 0.6 \mathrm{GHz}$ for resonant modes with an effective mode volume of only $V_{\text {eff }} \sim 7(\lambda / n)^{3}$ (where $V_{\text {eff }}$ is defined in terms of the peak electric energy density, as in Ref. 33, and has been calculated through finite-element simulations). Nevertheless, some degradation in the quality factors from those previously measured in the $1400 \mathrm{~nm}$ band is observed. These are believed to be at least in part due to absorption in the QD layers, as evidenced by the emission in the PL spectrum at these wavelengths [Fig. 3(a)].

\section{IMPROVED COLLECTION EFFICIENCY WITH FIBER TAPERS}

We now turn to the heart of the current work, which is a study of the gains in efficiency that can be achieved by using optical fiber tapers as a collection tool in PL measurements. This is initially done by comparing the amount of power obtained in free-space and fiber taper collection configurations, while maintaining identical free-space pumping conditions (in terms of pump-beam intensity and pump beam position). The free-space collection for a microdisk that has been pumped at normal incidence with $\sim 580 \mathrm{~W} / \mathrm{cm}^{2}$ at
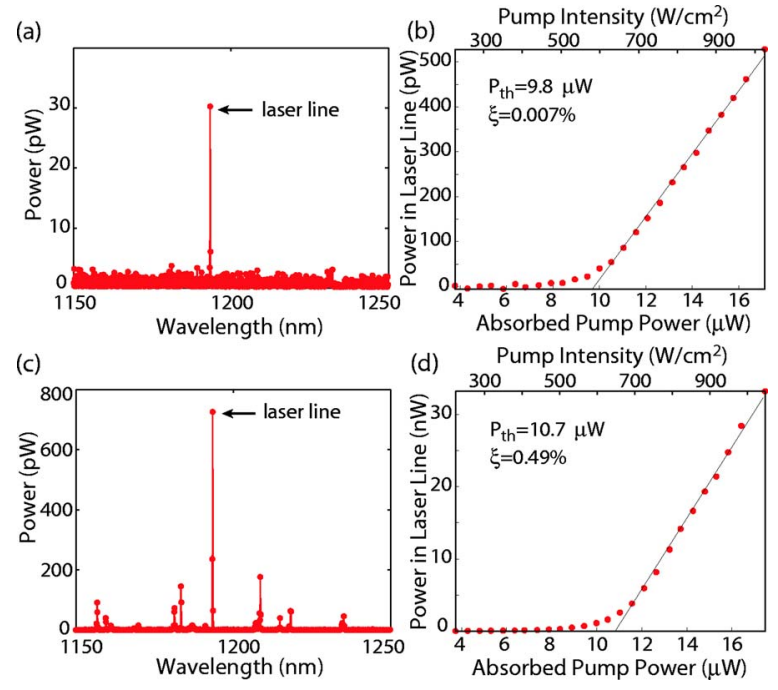

FIG. 4. (Color online) (a) Emission spectrum and (b) Light-invs-Light-out (L-L) curve for normal incidence free-space collection from a (free-space) optically pumped microdisk. Fiber taper collected (c) emission spectrum and (d) L-L curve from the same microdisk resonator, under identical pumping conditions. The emission spectra for both the free-space and fiber taper collection configurations [(a) and (c)] were taken near laser threshold at $\sim 580 \mathrm{~W} / \mathrm{cm}^{2}$ of incident pump beam intensity. The fiber taper collected power included that from the forward propagating transmission channel only. Unless otherwise specified, in all of the measurements described in this work, a detector sensitivity of $\sim 1 \mathrm{pW}$ is used, and the resolution bandwidth of the OSA is set to $0.1 \mathrm{~nm}$.

$830 \mathrm{~nm}$ is shown in Fig. 4(a). This pump intensity is near the laser threshold for this device (see below), and we see that the peak height at $\lambda \sim 1193.5 \mathrm{~nm}$ is $\sim 30 \mathrm{pW}$. For comparison to the fiber taper collection described below, an estimate of the optical losses in the free-space collection setup were made (after removal of the pump rejection filter). By measuring the collected pump laser power reflected off of the mirror-quality surface of the AlGaAs epitaxy, and assuming a $30 \%$ reflection coefficient from the AlGaAs surface, $43 \%$ of the reflected pump beam was collected into the OSA. Additional limitations in the normal incidence free-space collection stem from the finite numerical aperture of the collecting lens $(\mathrm{NA}=0.4)$ which covers only $4 \%$ of the full $4 \pi$ steradians.

Next, we consider the use of the optical fiber taper as a collection optic in the PL measurements. To obtain an estimate of the amount of coupling between the taper and the microdisk, the free-space pump beam is blocked, and passive measurements at wavelengths that are slightly red-detuned from the QD emission are performed as described above in Sec. III. Since the FSR for the low radial number WGMs of the microdisks studied here is $\sim 41 \mathrm{~nm}$ in the $1200 \mathrm{~nm}$ wavelength band, the modes coupled to and studied passively are typically a single FSR red-detuned from the lasing mode. For most experiments, the taper is placed in direct contact with the top edge of the microdisk, which increases the amount of coupling from that shown in Fig. 3(b) to transmission depths between $30 \%$ and $60 \%$. For this initial measurement, a resonance depth of $\sim 38 \%$ is obtained for a cav- 
ity mode at $\lambda \sim 1238.1 \mathrm{~nm}$, which gives us a qualitative estimate for the coupling to WGMs overlapping the peak of the gain spectrum..$^{51}$ This coupling depth corresponds to a taper collection efficiency $\eta_{0} \sim 11 \%$, where $\eta_{0}$ is defined ${ }^{19}$ as the fraction of the optical power from the cavity resonant mode that is coupled into the fundamental fiber taper mode in the forward propagating transmission direction. Other loss channels from the microdisk include intrinsic loss of the cavity in absence of the taper, parasitic coupling into higherorder, noncollected modes of the fiber taper, and for the standing wave modes studied here, coupling into the backwards propagating fundamental taper mode. For the moderate coupling depths measured here, the taper coupling efficiency into the forward and backward propagation directions is approximately equal, thus yielding an overall taper coupling efficiency of $\eta_{0}^{\prime} \sim 22 \%$ for this WGM.

Once this level of coupling has been achieved, the tunable laser output is blocked, the free-space pump beam is unblocked, and the output of the fiber taper is disconnected from the photodetector and connected to the OSA to measure the emitted power from the microdisk. Figure 4(c) shows the resulting spectrum collected by the fiber taper in the forward propagating transmission direction. We see that at the wavelength $\lambda \sim 1193.5 \mathrm{~nm}$, the peak height is $\sim 725 \mathrm{pW}$, which is nearly a factor of 25 times improvement over the peak height observed in normal incidence free-space collection. In addition, a significant amount of power is present within modes that were not detectable in the free-space case (the noise floor of the OSA was approximately $1 \mathrm{pW}$ ), due to the poor efficiency of collection in this configuration.

This straightforward comparison of the collected powers for a single pump power is not necessarily the most appropriate comparison, however. The reason for this is that the fiber taper loads the cavity, thus decreasing the $Q$ of the resonant modes and increasing the threshold pump power, so that for a given pump power the laser is not equally above threshold in the two measurements. Another, more appropriate comparison is the differential collection efficiency above threshold, which we label $\xi$. This is determined by measuring a light-in-light-out (L-L) curve for the microdisk and taking the slope of this curve above threshold. In these curves, the light-out is taken to be the total power within the laser line, while the light-in is taken to be the estimated absorbed pump power. The absorbed pump power is determined by multiplying the pump beam intensity by the area of the microdisk to get an incident pump power (the beam overlaps the entirety of the disk), and then multiplying this value by the absorption of the microdisk at $830 \mathrm{~nm}$. We estimate this absorption to be $\sim 10 \%$, assuming an absorption coefficient of $10^{4} \mathrm{~cm}^{-1}$ in the GaAs, quantum well, and QD layers, ${ }^{34}$ and a reflection coefficient of $30 \%$ at the GaAs-air interfaces at the top and bottom of the disk. The resulting L-L curves are shown in Figs. 4(b) and 4(d) for both free-space and fiber-taper collection. We see that the threshold pump power has indeed increased in the case of fiber-taper collection, but that the differential efficiency has also significantly improved and is more than 70 times that of the free-space value.

To study the tradeoffs between $\xi$ and threshold more closely, in Fig. 5(a) several L-L curves are plotted, each for a different taper position with respect to the microdisk (note (a)

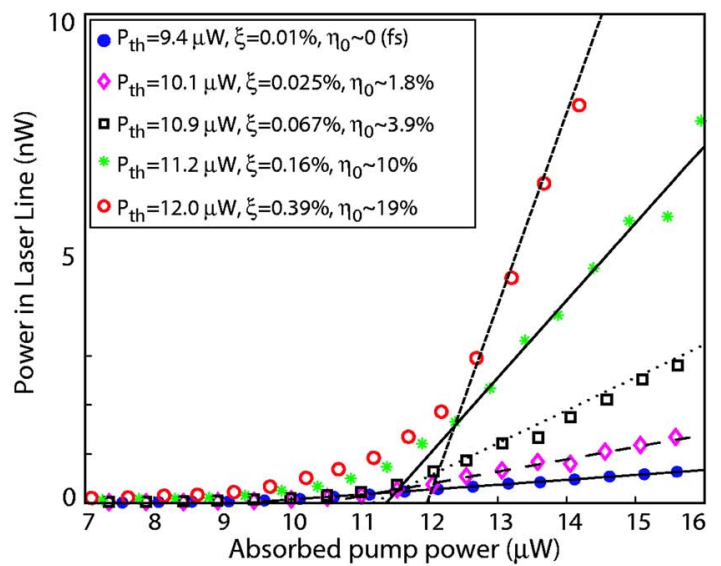

(b)

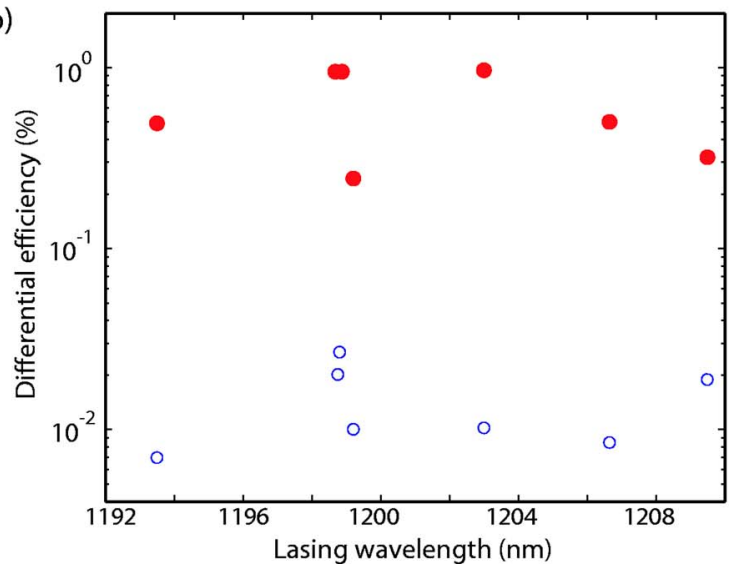

FIG. 5. (Color online) (a) L-L curves for free-space pumping and fiber taper collection at different taper positions. For each curve, we note the threshold pump power $\left(P_{\mathrm{th}}\right)$, the fiber taper collection efficiency $\left(\eta_{0}\right)$ for a mode that is red-detuned from the peak QD emission, and the above threshold differential efficiency $(\xi)$. (b) Scatter plot of the differential efficiency for fiber taper (filled circles) and free-space collection (open circles) for a number of different microdisk lasers. For these measurements the fiber taper collected power included that from the forward propagating transmission channel only.

that the microdisk studied here is not the same as the one studied above, but the qualitative behavior is identical). The different taper positions correspond to a varying level of coupling between the microdisk and taper, which we again qualitatively estimate through measurements of the coupling to a microdisk WGM that is red-detuned from the QD emission in the $1200 \mathrm{~nm}$ band. From Fig. 5(a), we see that in general, both the threshold power and $\xi$ increase with increasing $\eta_{0}$. As might be expected, in the course of these measurements it was possible in some cases to load the microdisk strongly enough to degrade the initial laser mode's $Q$ to the point that it no longer lases, and a different mode (with a higher loaded $Q$ ) begins to lase.

A number of different microdisk devices have been studied, and the results described above are fairly consistent from device to device, with $\xi$ routinely 1 to 2 orders of magnitude larger when fiber taper collection is employed. A scatter plot for some of this data is shown in Fig. 5(b). Despite the significant improvement obtained using the fiber taper, we see 
that the largest $\xi$ measured is roughly $10 \mathrm{nW} / \mu \mathrm{W}$, which means that only $1 \%$ of the pump photons are converted to a collected signal photon, and we should thus consider why $\xi$ is far below unity. We first note that when considering collection into both directions of the fiber taper, $\xi$ is actually $2 \%$ for the standing wave WGMs of the microdisks studied here. A measure of the fiber taper collection efficiency of the microdisk WGM laser light, $\eta_{0}$, from the passive wavelength scans described above indicate that the external fiber taper collection efficiency should be as high as $\sim 22 \%$ (corresponding to $11 \%$ for the forward transmission direction only). The total loss through the fiber taper and all of the fusion-splices and connections in the fiber path was measured to be $\sim 1.6 \mathrm{~dB}$, so that, if symmetric loss in the taper about the microdisk coupling region is assumed, $\sim 17 \%$ of the WGM laser photons collected by the taper are lost before they reach the OSA. Taken together, these two factors put an estimate of the upper bound on the fiber-coupled external laser efficiency of $18 \%$ for collection into both directions of the fiber.

The roughly order of magnitude difference between the measured $(2 \%)$ and expected $(18 \%)$ differential laser efficiency may be a result of several factors involving the complex dynamics within the DWELL active region. Previous measurements of DWELL injection lasers in stripe geometries ${ }^{22}$ indicate that the internal quantum efficiency of the quantum dots is $\eta_{i}^{Q D} \sim 0.5$ (this is roughly the percentage of carriers captured by the QDs in the DWELL structure that contribute to stimulated emission above threshold). This factor can certainly change from growth to growth, and given that the laser threshold values are roughly $2-2.5$ times higher than that measured in previous work on identically fabricated devices from a different wafer growth, ${ }^{21}$ we might qualitatively expect $\xi$ to be reduced by a factor of $\sim 4$ to 5 due to $\eta_{i}^{Q D}$.

Both the spectral and spatial distribution of carriers within the microdisk may also lead to reductions in the laser differential efficiency through incomplete clamping of the spontaneous emission into the nonlasing modes of the microdisk above threshold. To examine such effects in our structures, we measure L-L curves [Fig. 6(a)] for a number of the most prominent non-lasing WGMs of the microdisk studied in Fig. 4. We see that the emission into these modes is largely clamped above the threshold for the lasing mode (estimated to be $10.7 \mu \mathrm{W}$ of absorbed pump power). The aggregate effect is clearly seen in Fig. 6(b), where the power into the nonlasing WGMs has been summed and plotted along with the L-L curve for the lasing mode. Such clamping has been reported by other authors for similarly sized microdisks, ${ }^{35}$ while smaller microcavity devices with a larger laser mode spontaneous emission rate have exhibited a gradual rollover and/or incomplete clamping of spontaneous emission. ${ }^{7,35-37}$ Measurement of the background spontaneous emission into non-WGM, radiation modes of the microdisk was performed using free-space collection (the fiber taper is much more sensitive to WGM emission than to emission from the center of the microdisk into radiation modes), and did show incomplete clamping of the spontaneous emission. This sort of spatial hole burning has been predicted in numerical modeling of microdisk cavities. ${ }^{38}$ If this is the case, the effective pump
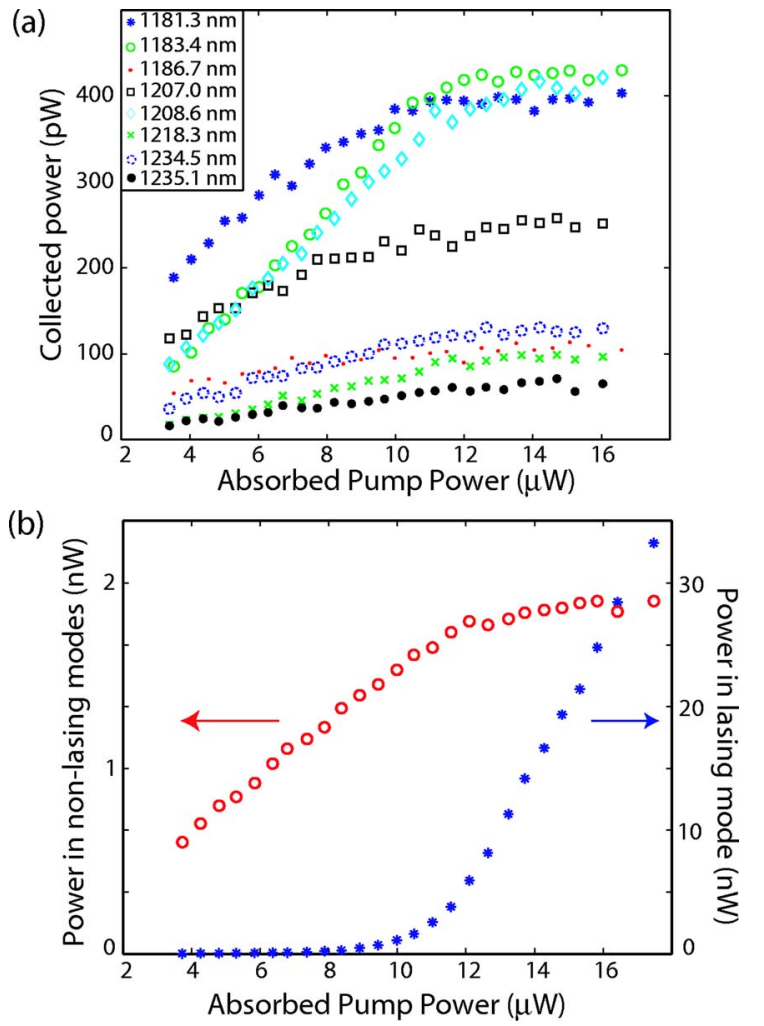

FIG. 6. (Color online) (a) L-L curves for nonlasing modes of the disk studied in Fig. 4. (b) Total power in the nonlasing modes (shown by open circles), showing saturation for pump values close to the threshold value for the lasing mode (shown by asterisks). Note that the $y$ axis scale for the lasing mode is 15 times larger than that for the nonlasing modes.

area is limited to a region about the WGM. Assuming that the WGM radial width is approximately $\left(\lambda / n_{\text {eff }}\right)$, where $n_{\text {eff }}$ is the effective refractive index in the plane of the microdisk, ${ }^{52}$ this corresponds to a $7 \mu \mathrm{m}^{2}$ area in the devices under test here. Since the total disk area is $\sim 16 \mu \mathrm{m}^{2}$, then only $7 / 16$ of the pump photons would be effectively pumping the WGM. Including this factor brings the expected value of $\xi$ within the range of experimentally measured values.

Aside from reducing taper loss (loss $<0.5 \mathrm{~dB}$ can be easily achieved in our lab), $\eta_{0}$ is the main parameter that can be improved upon to increase $\xi$. This can be done by adjusting the geometry of the disk (using thinner disks, for example) to bring the effective index of the WGMs of the semiconductor microdisks closer to that of the silica fiber taper, so that more efficient coupling can be obtained. A study of such modifications in Si microdisk structures has been undertaken, and the regimes of critical coupling and overcoupling have been achieved. ${ }^{26}$ In addition, if spatial hole burning is significant, another factor that could potentially be improved is the method of pumping. In particular, the pumping beam could be shaped to preferentially pump the perimeter of the microdisk (i.e., an annular-shaped beam could be used). Alternately, as discussed below, a fiber taper could be used to pump the microdisk. 


\section{FIBER-PUMPED MICRODISK LASERS}

In addition to improving the collection efficiency, optical fiber tapers have the potential for improving the pump efficiency of these QD-containing microdisks; such an effect has in fact been demonstrated in previous work on doped glass microcavities. ${ }^{39-41}$ In particular, if the pump laser is resonant with a WGM of the microdisk, light can be absorbed with high efficiency, and in the case of critical coupling, complete power transfer can be achieved. This should be contrasted with the case of free-space pumping, where only a small percent $(10 \%$ for the devices we have considered here) of the incident pump light is absorbed by the device, and some of this absorption is in a region (the center of the microdisk) that does not contribute to useful gain for the resonant WGMs. ${ }^{38}$

For an initial demonstration, we use a tunable laser operating in the $980 \mathrm{~nm}$ band as a pump source. The $830 \mathrm{~nm}$ pump laser is not used because the absorption within the microdisk at this wavelength is too large to allow uniform pumping of the microdisk perimeter, as the pump light is absorbed before a single round trip around the cavity can be made. At $980 \mathrm{~nm}$, the material absorption is still relatively high (the quantum well layer will be highly absorbing), so that the $Q_{980}$ of WGM modes near the pump wavelength are not expected to exceed a few hundred. The pump laser is connected to the fiber taper input, and the fiber taper output is connected to the OSA. The taper is contacted to the side of the microdisk, and the pump wavelength and polarization are manually adjusted until the collected power in the OSA is maximized (this is necessary in order to resonantly couple to a mode within the pump wavelength band). A typical L-L curve and sub-threshold spectrum are shown in Fig. 7. We see that a significant amount of power is collected into the fiber taper, and that in particular, the sub-threshold spectrum shows a number of well-resolved modes with a good signalto-noise ratio. The estimated absorbed pump power in the microdisk displayed in Fig. 7(a) corresponds to $66 \%$ of the input power in the fiber taper, and is found by taking the difference in the $980 \mathrm{~nm}$ band taper transmission between when the taper is displaced tens of microns above the microdisk (no coupling) and when it is in contact with the microdisk (strongly coupled). We note that the pump threshold value in this pumping geometry is only about a factor of two less than the incident pump power in the $830 \mathrm{~nm}$ free-space pumping, and is significantly larger than what might be expected (ideally, the pump power here should be less than the absorbed pump power in the $830 \mathrm{~nm}$ pumping). This is most likely a result of the relatively crude method we have employed to estimate the power absorbed in the microdisk; a much more accurate method for determining the coupled pump power uses the wavelength-dependent transmission of the fiber taper to map out the resonance line due to the WGM at the pump wavelength. Here, the strong absorption of the microdisk in the $980 \mathrm{~nm}$ band makes it difficult to separate resonantly coupled power from scattering losses at the tapermicrodisk junction. In order to more carefully study the efficiency of this fiber-pumping and fiber-collecting configuration, experiments in which an excited state of the quantum dots is resonantly pumped through the fiber taper are currently underway. (a)

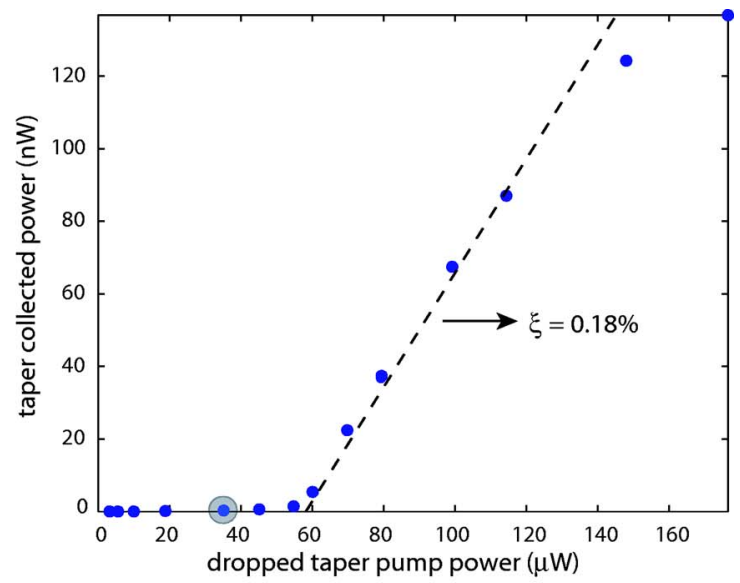

(b)

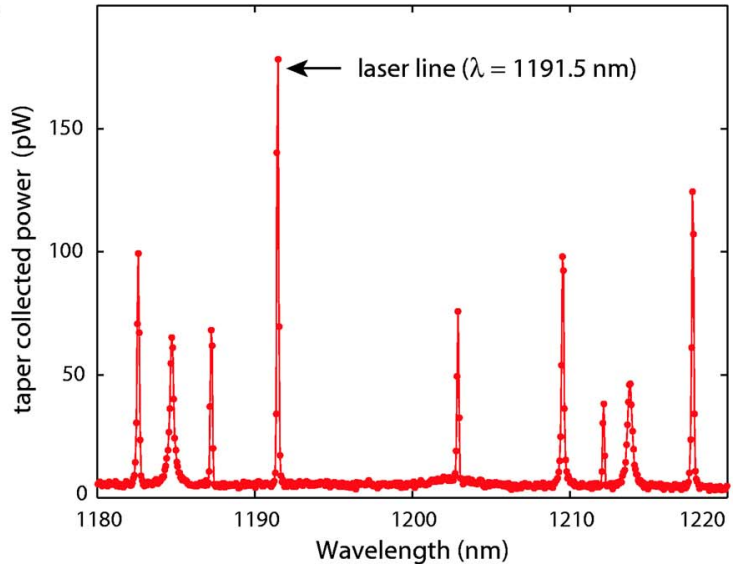

FIG. 7. (Color online) (a) L-L curve for a QD-microdisk device where the fiber taper is used for both pumping (at $\lambda=967.6 \mathrm{~nm}$ ) and collection. The absorbed power was estimated to be $66 \%$ of the input power in the fiber taper. (b) Sub-threshold spectrum for this device taken at an estimated absorbed power of $37 \mu \mathrm{W}$ [highlighted point in (a)].

\section{DISCUSSION AND FUTURE APPLICATIONS}

As mentioned in the introduction of this paper, efficient optical access to wavelength-scale microcavities is of great importance to quantum optics and information processing applications currently being investigated within cavity QED systems. In almost any application involving the coherent transfer or manipulation of quantum information, loss is a significant detriment. As described in Ref. 42, current implementations of linear optics quantum computing require a near-unity collection efficiency of emitted photons from a single photon source. The same is true for applications involving quantum repeaters in a quantum network. ${ }^{43} \mathrm{~A}$ solution that is often proposed is to embed the single photon emitter within a microcavity with a high spontaneous emission coupling factor $\beta$, so that the majority of emitted photons are coupled into the microcavity mode. However, it is important to note that even for a $\beta=1$ microcavity, it is still necessary to have a method to effectively collect all of the photons that are radiated by that one cavity mode. ${ }^{44}$ Also, from a very practical perspective, efficient collection of emitted light from a microcavity is of premium importance for optical telecommunication wavelengths $>1 \mu \mathrm{m}$, where the 
dark count rates from single photon counters are often 2 to 3 orders of magnitude larger than the Si single photon counters used at shorter wavelengths. ${ }^{45}$

An efficient coupling channel can also enable a number of different types of experiments. Having access to this coupling channel makes the cavity transmission (and reflection) an experimentally accessible parameter whose behavior can be monitored to detect signatures of specific types of system behavior. In recent experimental measurements of coupling between a single quantum dot and a resonant mode of a semiconductor microcavity, 5,6,30,32,46 spontaneous emission from the coupled system is the only parameter measured. Alternatively, using fiber tapers, vacuum-Rabi splitting can be detected by simply measuring the transmission through the cavity as a function of the input wavelength to the cavity; such an experiment is directly analogous to the experiments done with cooled alkali atoms coupled to a Fabry-Perot cavity. ${ }^{47,48}$ Nonlinear effects, such as optical bistability and photon blockade, and coherent control of the quantum system are also more easily observed through the optical transmission or reflection channel of a microcavity. Perhaps most importantly, by knowing the precise level of coupling between the fiber taper and the microcavity, the number of photons injected into the cavity can be precisely calibrated. This is obviously of paramount importance in experiments that involve few or single cavity photons. Finally, we note that although many of the advantages we have described are also applicable to in-plane waveguides that are microfabricated next to the cavities, the fiber taper provides a level of flexibility that, for example, allows for rapid device characterization, as described in detail in Ref. 49.

In conclusion, we have demonstrated the utility of fiber tapers in the photoluminescence measurements of QDcontaining microdisk cavities. Improvements in collection efficiency of 1 to 2 orders of magnitude in comparison to normal incidence free-space measurements are exhibited, and initial measurements on microdisk lasers employing fiber taper pumping and collection have been presented. The differential laser efficiency was measured to be as high as $2 \%$ when accounting for collected light into both directions of the fiber taper. From direct passive measurements of the microdisks at wavelengths $30 \mathrm{~nm}$ red detuned from the ground-state QD emission peak, the fiber taper collection efficiency of light in a microdisk whispering gallery mode is estimated to be $18 \%$ (including both directions of emission within the fiber taper and all optical losses in the system). With further modifications of the microdisk structure to enable better phase-matching of the fiber taper waveguide and the whispering-gallery modes of the high-refractive index microdisk, ${ }^{26}$ significant increases in coupling efficiency, approaching unity, are believed possible with this technique.

\section{ACKNOWLEDGMENTS}

The authors thank Matt Borselli, Paul Barclay, and Tom Johnson for valuable discussions and assistance in setting up some of the measurement equipment, and Matt Borselli for numerical simulations of the microdisk modes. K.S. thanks the Hertz Foundation for its graduate fellowship support.

\section{APPENDIX: DESCRIPTION OF THE EXPERIMENTAL SETUP}

The experimental setup shown in Fig. 2 is comprised of a taper probing station that has been integrated into a PL measurement setup. The taper probing station consists of a motorized $X-Y$ stage (50 nm encoded resolution) on which the microcavity chip is placed, while the fiber taper waveguide is held in a "u"-shaped configuration on an acrylic mount as shown in Fig. 2(b). The acrylic fiber mount is attached to a separate motorized $z$ axis stage (50 $\mathrm{nm}$ encoded resolution) so that the fiber taper can be precisely aligned to the microdisk (the taper moves vertically and the sample moves inplane). Passive measurements of the resonator modes are performed by connecting the input of the fiber taper to a $1200 \mathrm{~nm}$ band scanning tunable laser, with the polarization of the laser output at the taper-microdisk interaction region controlled using a paddle wheel polarization controller. The entire taper probing setup (motorized stages, microcavity chip, and fiber taper waveguide) is mounted onto a larger manually actuated $X-Y-Z$ stage that is positioned underneath an ultra-long working distance objective lens $(\mathrm{NA}=0.4)$. This microscope objective is part of a PL setup that provides normal incidence pumping and free-space collection from the samples. The pump laser in the majority of the measurements is a $830 \mathrm{~nm}$ laser diode that is operated in quasicontinuous-wave operation (280 ns pulse width, $300 \mathrm{~ns}$ period). The pump beam is shaped into a Gaussian-type profile by sending the laser beam through a section of single mode optical fiber, and is then focused onto the sample with a spot size that is slightly larger than the size of the microdisk (area $\sim 18 \mu \mathrm{m}^{2}$ ). The free-space pump laser power is monitored by using a $830 \mathrm{~nm}$ wavelength 50/50 nonpolarizing beamsplitter (NPBS) with a calibrated photodetector (PD) on one of the ports. The QD free-space photoluminescence in the $1200 \mathrm{~nm}$ band is collected at normal incidence from the sample surface using the same objective lens for pump focusing, is transmitted through the $830 \mathrm{~nm}$ NPBS and a longpass pump rejection filter (LPF), and is finally collected into a multimode fiber (MMF) using an objective lens with $\mathrm{NA}=0.14$. The luminescence collected by this $\mathrm{MMF}$ is wavelength resolved by a Hewlett Packard 70452B optical spectrum analyzer. For fiber taper measurements, the fiber taper is strung across the sample and positioned in the nearfield of the microdisk from above, allowing simultaneous (normal incidence) free-space and fiber taper optical pumping and photoluminescence collection. The output of the fiber taper can either be connected to an InGaAs photodetector (PD) for wavelength scans using the tunable laser source, or to the OSA for analysis of the photoluminescence from the microdisk. Alignment of the pump beam and the fiber taper to the microdisk is performed by imaging through the pump and collection objective lens, as shown in the inset (a mirror flips in-and-out of the free-space photoluminescence beam path to direct the image to a $12 \times$ zoom imaging system). All of the measurements presented here were performed with the sample maintained in a room temperature environment, with no active cooling or temperature control. 
*Electronic address: kartik@caltech.edu

${ }^{1}$ Y. Yamamoto and R. E. Slusher, Phys. Today 46 (6), 66 (1993).

${ }^{2}$ H. Mabuchi and A. C. Doherty, Science 298, 1372 (2002).

${ }^{3}$ H. J. Kimble, Phys. Scr., T T76, 127 (1998).

${ }^{4}$ W. L. Barnes, G. Björk, J. Gérard, P. Jonsson, J. Wasey, P. Worthing, and V. Zwiller, Eur. Phys. J. D 18, 197 (2002).

${ }^{5}$ G. S. Solomon, M. Pelton, and Y. Yamamoto, Phys. Rev. Lett. 86, 3903 (2001).

${ }^{6}$ J.-M. Gérard and B. Gayral, J. Lightwave Technol. 17, 2089 (1999).

${ }^{7}$ R. E. Slusher, Semicond. Sci. Technol. 9, 2025 (1994).

${ }^{8}$ A. Levi, R. Slusher, S. McCall, J. Glass, S. Pearton, and R. Logani, Appl. Phys. Lett. 62, 561 (1993).

${ }^{9}$ J. Nöckel, Phys. Status Solidi A 188, 921 (2001).

${ }^{10}$ K. Srinivasan, P. E. Barclay, M. Borselli, and O. Painter, Phys. Rev. B 70, 081306(R) (2004).

${ }^{11}$ T. A. Birks, and Y. W. Li, J. Lightwave Technol. 10, 432 (1992).

${ }^{12}$ T. Birks, in Optical Fiber Communications Conference (OFC) (2004).

${ }^{13}$ J. Knight, G. Cheung, F. Jacques, and T. Birks, Opt. Lett. 22, 1129 (1997).

${ }^{14}$ N. Dubreuil, J. C. Knight, D. K. Leventhal, V. Sandoghdar, J. Hare, and V. Lefèvre, Opt. Lett. 20, 813 (1995).

${ }^{15}$ M. Cai, O. Painter, and K. J. Vahala, Phys. Rev. Lett. 85, 74 (2000).

${ }^{16}$ S. M. Spillane, T. J. Kippenberg, O. J. Painter, and K. J. Vahala, Phys. Rev. Lett. 91, 043902 (2003).

${ }^{17}$ D. K. Armani, T. J. Kippenberg, S. M. Spillane, and K. J. Vahala, Nature (London) 421, 925 (2003).

${ }^{18}$ P. E. Barclay, K. Srinivasan, M. Borselli, and O. Painter, Opt. Lett. 29, 697 (2004).

${ }^{19}$ P. E. Barclay, K. Srinivasan, and O. Painter, Opt. Express 13, 801 (2005).

${ }^{20}$ M. Borselli, K. Srinivasan, P. Barclay, and O. Painter, Appl. Phys. Lett. 85, 3693 (2004).

${ }^{21}$ K. Srinivasan, M. Borselli, T. Johnson, P. Barclay, O. Painter, A. Stintz, and S. Krishna, Appl. Phys. Lett. 86, 151106 (2005).

${ }^{22}$ G. Liu, A. Stintz, H. Li, T. Newell, A. Gray, P. Varangis, K. Malloy, and L. Lester, IEEE J. Quantum Electron. 36, 1272 (2000)

${ }^{23}$ B. Gayral, J. M. Gérard, A. Lemaître, C. Dupuis, L. Manin, and J. L. Pelouard, Appl. Phys. Lett. 75, 1908 (1999).

${ }^{24}$ P. Michler, A. Kiraz, L. Zhang, C. Becher, E. Hu, and A. Imamoglu, Appl. Phys. Lett. 77, 184 (2000).

${ }^{25}$ C. Manolatou, M. Khan, S. Fan, P. R. Villeneuve, H. Haus, and J. Joannopoulos, IEEE J. Quantum Electron. 35, 1322 (1999).

${ }^{26} \mathrm{M}$. Borselli, T. Johnson, and O. Painter (unpublished).

${ }^{27}$ D. S. Weiss, V. Sandoghdar, J. Hare, V. Lefèvre-Seguin, J.-M. Raimond, and S. Haroche, Opt. Lett. 20, 1835 (1995).

${ }^{28}$ T. Kippenberg, S. Spillane, and K. Vahala, Opt. Lett. 27, 1669 (2002).

${ }^{29}$ S. M. Spillane, T. J. Kippenberg, K. J. Vahala, K. W. Goh, E.
Wilcut, and H. J. Kimble, Phys. Rev. A 71, 013817 (2005).

${ }^{30}$ T. Yoshie, A. Scherer, J. Hendrickson, G. Khitrova, H. Gibbs, G. Rupper, C. Ell, Q. Schenkin, and D. Deppe, Nature (London) 432, 200 (2004).

${ }^{31}$ A. Loffler, J. Reithmaier, G. Sek, C. Hofmann, S. Reitzenstein, M. Kamp, and A. Forchel, Appl. Phys. Lett. 86, 111105 (2005).

${ }^{32}$ A. Badolato, K. Hennessy, M. Atature, J. Dreiser, E. Hu, P. Petroff, and A. Imamoglu, Science 308, 1158 (2005).

${ }^{33}$ L. C. Andreani, G. Panzarini, and J.-M. Gérard, Phys. Rev. B 60, 13276 (1999).

${ }^{34}$ L. A. Coldren and S. W. Corzine, Diode Lasers and Photonic Integrated Circuits (Wiley, New York, NY, 1995).

${ }^{35}$ R. E. Slusher, A. Levi, U. Mohideen, S. McCall, S. Pearton, and R. Logan, Appl. Phys. Lett. 63, 1310 (1993).

${ }^{36}$ M. Fujita, R. Ushigome, and T. Baba, IEEE Photonics Technol. Lett. 13, 403 (2001).

${ }^{37}$ K. Srinivasan, P. E. Barclay, O. Painter, J. Chen, A. Y. Cho, and C. Gmachl, Appl. Phys. Lett. 83, 1915 (2003).

${ }^{38}$ K. Nozaki and T. Baba, IEEE J. Sel. Areas Commun. 23, 1411 (2005).

${ }^{39}$ V. Sandoghdar, F. Treussart, J. Hare, V. Lefèvre-Seguin, J.-M. Raimond, and S. Haroche, Phys. Rev. A 54, R1777 (1996).

${ }^{40}$ M. Cai, O. Painter, K. Vahala, and P. Sercel, Opt. Lett. 25, 1430 (2000).

${ }^{41}$ L. Yang, D. Armani, and K. Vahala, Appl. Phys. Lett. 83, 825 (2003).

${ }^{42}$ A. Kiraz, M. Atature, and A. Immamoglu, Phys. Rev. A 69, 032305 (2004).

${ }^{43}$ J. I. Cirac, P. Zoller, H. J. Kimble, and H. Mabuchi, Phys. Rev. Lett. 78, 3221 (1997).

${ }^{44}$ J. M. Gérard and B. Gayral, Proc. SPIE 5361, 88 (2004).

${ }^{45}$ G. Ribordy, N. Gisin, O. Guinnard, D. Stucki, M. Wegmuller, and H. Zbinden, J. Mod. Opt. 51, 1381 (2004).

${ }^{46}$ J. Reithmaier, G. Sek, A. Loffer, C. Hoffman, S. Kuhn, S. Reitzenstein, L. Keldysh, V. Kulakovskii, T. Reinecke, and A. Forchel, Nature (London) 432, 197 (2004).

${ }^{47}$ R. I. Thompson, G. Rempe, and H. J. Kimble, Phys. Rev. Lett. 68, 1132 (1992).

${ }^{48}$ A. Boca, R. Miller, K. M. Birnbaum, A. D. Boozer, J. McKeever, and H. J. Kimble, Phys. Rev. Lett. 93, 233603 (2004).

${ }^{49}$ K. Srinivasan, P. Barclay, M. Borselli, and O. Painter, IEEE J. Sel. Areas Commun. 23, 1321 (2005).

${ }^{50}$ The WGMs in the $1400 \mathrm{~nm}$ wavelength band are expected to have very similar radiation and scattering losses as those in the $1200 \mathrm{~nm}$ band for the microdisk geometries studied here. Differences in $Q$ at these two wavelengths are thus expected to be indicative of wavelength-dependent material absorption losses.

${ }^{51}$ The coupling between the taper and microdisk can be different for different cavity modes, so this technique is used primarily as a qualitative guide.

${ }^{52}$ Finite-element simulations have shown this to be an accurate estimate. 